\section{Sistema Activador del Plasminógeno en la Enfermedad Periodontal}

\author{
Plasminogen Activator System in Periodontal Disease
}

\begin{abstract}
Resumen
El sistema activador del plasminógeno participa activamente en la dinámica de la enfermedad periodontal y se encuentra finamente compensado en cuanto a sus activadores e inhibidores, aunque existen periodos donde las bolsas periodontales reagudizadas pueden romper el fino equilibrio entre activadores ( $\mathrm{t}-\mathrm{PA}$ ) e inhibidores del plasminógeno (PAI-2) desencadenando el progreso de la enfermedad periodontal; cabe destacar la participación de patógenos bacterianos como la Porphyromona gingivalis, que se caracteriza por generar el desbalance entre los principales activadores e inhibidores con el consecuente incremento de la actividad fibrinolítica. Los efectos del desbalance de los constituyentes del sistema activador del plasminógeno, en particular t-PA, toma mayor relevancia en los eventos hemorrágicos a nivel gingival de pacientes con deficiencias de los factores de la coagulación como los anticoagulados por warfarina y enfermedad hepática severa como la cirrosis. El sistema activador del plasminógeno como parte del sistema fibrinolítico es descrito según la literatura más relevante en cuanto a su participación en la patogénesis de la enfermedad periodontal y sus componentes son descritos comparativamente a nivel local o periodontal con el nivel sistémico.

Palabras clave: periodontitis, fibrinolisis, activador del plasminógeno tisular, inhibidor del activador del plasminógeno 2
\end{abstract}

\begin{abstract}
The plasminogen activator system is actively involved in periodontal disease dynamics and is finely balanced in terms of their activators and inhibitors, although there are periods where exacerbation of periodontal pockets can break the delicate balance between activators ( $\mathrm{t}-\mathrm{PA})$ and inhibitors of plasminogen (PAI-2) triggering the progression of periodontal disease include the participation of bacterial pathogens Porphyromonas gingivalis as characterized by generating the major imbalance between activators and inhibitors with a consequent increase in fibrinolytic activity. The effects of the imbalance of the constituents of plasminogen activator system in particular t-PA takes on more importance in the gingival level bleeding events in patients with deficiencies of coagulation factors such as warfarin anticoagulation or severe liver disease such as cirrhosis. The plasminogen activator system as part of the fibrinolytic system is described by the relevant literature regarding their involvement in the pathogenesis of periodontal disease and its components are described comparatively local or periodontal level with systemic level.
\end{abstract}

Key words: periodontitis, fibrinolysis, tissue plasminogen activator, plasminogen activator inhibitor 2
Artículo de Revisión

\section{* Christian Méndez Visag}

* Cirujano Dentista egresado de la Facultad de Odontología de la UNMSM.

\title{
Correspondencia:
}

Christian Méndez Visag

Facultad de Odontología

Correo electrónico: c66mendez@hotmail.com

Fecha de recepción: 23-11-11

Fecha de aceptación: 12-07-12

\section{Generalidades sobre el Sistema Activador del Plasminógeno}

El Sistema Activador del Plasminógeno (SAP) es el responsable de la degradación de coágulos sanguíneos intravasculares, mientras que contribuye también en la proteolisis extracelular en una amplia variedad de procesos fisiológicos (ej.: remodelación tisular, migración celular, curación de heridas, angiogenesis, implantación trofoblástica, ovulación y desarrollo fetal) y patológicos (ej.: inflamación crónica y aguda, preclampsia, retardo del desarrollo intrauterino, invasión tumoral y metástasis). ${ }^{1,2,3}$
Actualmente se conocen cuatro reguladores principales del SAP: Activador del plasminógeno tipo tisular (t-PA), Activador del plasminógeno tipo uroquinasa (u-PA), Inhibidor del Activador del Plasminógeno-1 (PAI-1), Inhibidor del Activador del Plasminógeno-2 (PAI-2). Cada uno de ellos cumple una función específica o especializada en regular la conversión del plasminógeno a plasmina, siendo la plasmina una proteasa relacionada a tripsina que degrada fibrina y diversas proteínas de matriz extracelular y de adhesión (ej.: proteoglicanos, lamininas, fibronectina y vitronectina), así como también elementos de la sangre como fibrinógeno, factor V, Factor VIII, protrombina y factor XII y activa procolagenasas, contribuyendo así a la degradación y recambio de la matriz extracelular. Por lo tanto, siempre que se forme plasmina en un coágulo de sangre, este puede lisar el coágulo y destruir muchos de los factores de coagulación, provocando hipocoagulabilidad de la sangre. ${ }^{1,4}$

\section{Activadores del Plasminógeno ( $t$-PA y u-PA)}

- Activador del plasminógeno tipo tisular (t-PA)

Es una proteasa de serina considerado como el principal activador fibrinolítico por su elevada afinidad a fibrina y es un mal activador del plasminógeno 
cuando no está presente la fibrina. El t-PA se enlaza a la fibrina y activa al plasminógeno unido con una rapidez varios cientos de veces mayor que al plasminógeno en circulación. En situaciones fisiológicas su concentración media en plasma es de $5 \mathrm{ng} / \mathrm{ml}^{5}$, la especificidad del t-PA a la fibrina limita la formación sistémica de plasmina y la inducción de un estado lítico sistémico. Es producido por diversos tipos de células, entre ellos las células endoteliales y epiteliales, macrófagos y fibroblastos $6,7,8,9$, su depuración es primordialmente por metabolismo hepático y su vida media es de cinco a diez minutos. ${ }^{6}$

\section{- Activador del plasminógeno tipo uroquinasa (u-PA)}

El u-PA se puede aislar de células del riñón humano en cultivo. Se encuentra predominantemente unido a membrana y está relacionada a la migración de células y proteolisis pericelular en situaciones fisiológicas y patológicas ${ }^{10}$, su concentración media en plasma es de $0.2 \mathrm{ng} / \mathrm{ml}^{5}$, puede ser producido por diversos tipos de células entre ellas las células endoteliales, epiteliales, macrófagos y fibroblastos. ${ }^{6,7.8 .9}$

Inhibidores de los Activadores del Plasminógeno (PAI-1 y PAI-2)

- Inhibidor del Activador del Plasminógeno-1 (PAI-1)

El PAI-1 es sintetizado por diversos tipos de células como hepatocitos en cultivo, células endoteliales, entre otras. Pero está más relacionado a células endoteliales y también es encontrado en los gránulos alfa de plaquetas sanguíneas.

Del total de proteínas secretadas por células endoteliales en cultivo, el PAI-1 representa el $12.5 \%$. La concentración media de este inhibidor en plasma es de $5 \mathrm{ng} / \mathrm{ml} .{ }^{5}$ La vida media del t-PA en plasma debido a la inactivación por alfa 2- antiplasmina es de 90 a $180 \mathrm{mi}$ nutos, mientras que la inactivación de t-PA se da en la escala de segundos.

El PAI-1 reacciona con ambos u-PA y $\mathrm{t}-\mathrm{PA}$, pero con mayor especificidad para el t-PA. ${ }^{2,3,5,7}$

\section{- Inhibidor del Activador del Plas- minógeno-2 (PAI-2)}

Miembro de la familia Serpin (Inhibidores de Proteasa de Serina) se encuentra bajo la forma no glicosilada o de bajo peso molecular BMP (47 KD) y en la forma glicosilada o de elevado peso molecular EPM (60 KD). Al respecto, se ha demostrado una diferencia en su localización según el estado de glicolización, siendo el PAI-2 de BMP intracelular y el PAI -2 de EPM secretado, pero comparten similares características de inhibición, aunque existe controversia por estudios recientes. PAI-2 es un eficiente inhibidor de u-PA, pero es más lento que PAI-1 (por un factor de $1 \mathrm{a}$ 5 veces, respectivamente) para inhibir al t-PA de doble cadena. Comparado con PAI-1 este es un pobre inhibidor de t-PA de cadena única, lo que hace pensar que PAI-2 no contribuye adecuadamente a la inhibición del t-PA de cadena única in vivo.

La activación del plasminógeno por u-PA ocurre preferencialmente en la superficie celular a través de la unión u-PA y plasminógeno a receptores específicos. El u-PA unido al receptor es todavía eficientemente inhibido por PAI-2 y PAI-1. Su concentración media en plasma es inferior a $5 \mathrm{ng} / \mathrm{ml}$ pero se incrementa hasta 50 veces en mujeres al término de la gestación. ${ }^{1,2} \mathrm{La}$ expresión de PAI-2 se pensaba limitado a macrófagos/monocitos y a la placenta, pero recientemente se encontró en una variedad de tipos celulares en los que es sobreexpresado por estímulos inflamatorios.

\section{Regulación}

La expresión tanto de activadores como de inhibidores está regulado por una amplia variedad de factores que pueden actuar según el tipo celular y el estado de diferenciación celular. Además, la interacción entre varias señales de transducción pueden ser sinérgicos o antagónicos. Así se tiene las citocinas proinflamatorias FNT-alfa e IL-1beta con efecto estimulatorio. Este efecto que es potenciado por AINES, como la indometacina y naproxeno; mientras que el efecto inhibitorio se obtiene con FNT-beta y dexametasona. ${ }^{1}$

\section{Sistema activador del plasminógeno y Estado Periodontal}

Constituyentes del SAP y su participación en la enfermedad periodontal

La periodontitis es un proceso inflamatorio local que media la destrucción del tejido periodontal por agresión bacteriana. Mientras que la respuesta del huésped a nivel local está mediada por citocinas y productos inflamatorios que exacerban la injuria tisular.
El fluido crevicular gingival (FCG) se sabe ahora que es un buen medio para proveer información útil del estado y grado de deterioro periodontal. El proceso destructivo del tejido periodontal está asociado con niveles incrementados de enzimas proteolíticas en el FCG y dentro del arsenal de agentes proinflamatorios tenemos a los activadores del plasminógeno ( $\mathrm{t}-\mathrm{PA}$ y u-PA) que son proteasas de serina, los cuales forman parte de la compleja cascada de enzimas involucradas en la fibrinolisis. ${ }^{8}$ A la vez la plasmina y los activadores del plasminógeno son regulados extracelularmente a través de inhibidores de proteinasas entre ellos: alfa 2-macroglobulina, alfa 1-inhibidor proteinasa, alfa 2-antiplasmina, inhibidor del activador del plasminógeno-1 (PAI-1) y el Inhibidor del activador del plasminógeno-2(PAI-2).

\section{Activadores del plasminógeno tipo ti- sular y uroquinasa en la enfermedad periodontal}

La plasmina puede ser formada localmente en el sitio de la inflamación por proteolisis limitada del precursor inactivo, el plasminógeno. En el tejido periodontal enfermo las células responsables de la sobrerregulación de los activadores del plasminógeno tipo tisular y uroquinasa son los queratinocitos del epitelio sulcular y de unión, así como por fibroblastos gingivales, macrófagos y células endoteliales. $5,7,8,9,10$

Pero existe una significativa diferencia en la expresión de los PA en el FCG de muestras del tejido periodontal con periodontitis, siendo el t-PA encontrado en elevadas concentraciones, inclusive muy superiores a los niveles plasmáticos 5,7,8,9,11,12,13 con valores promedio de $730 \mathrm{ng} / \mathrm{ml}$, que equivale aproximadamente 150 veces los niveles plasmáticos, mientras que la concentración media del u-PA fue de alrededor de los $100 \mathrm{ng} / \mathrm{ml}$ lo que equivale a 500 veces la concentración plasmática aproximadamente; pero esta elevada concentración del u-PA en el FCG en términos de molaridad equivale a solo el $20 \%$ de la concentración de t-PA. ${ }^{5}$

La actividad t-PA libre en el FCG detectado por zimografia estuvo en el rango de 50-100 pg/ul, mientras que exámenes de ELISA evaluaron ambas formas libres y complejas de t-PA, los cuales revelan concentraciones promedio de 1,6 ng/ul del antígeno total de t-PA en el FCG. De esta forma la actividad enzimática observada por zimografía representó solo una pequeña fracción del activador plasminógeno total pre- 
sente en las muestras. Según un estudio la concentración total de antígeno t-PA medido por ELISA (1,6 ng/ul) fue 200 veces más elevado en el FCG que los valores plasmáticos normales del t-PA. ${ }^{14}$

Además, mediante escalas semicuantitativas se demostró la abundante distribución de ARNm y antigenicidad para el t-PA en el epitelio sulcular y epitelio de unión. ${ }^{7}$

Por otro lado, se ha demostrado que los fibroblastos gingivales en medios de cultivo pueden ser estimulados por proteasas del Porphyromonas gingivalis y Porphyromonas endodontalis para secretar valores incrementados de colagenasas y el activador del plasminógeno (t-PA), adicionalmente la plasmina activada juega un rol en el progreso de la inflamación del tejido periodontal a través de la activación de metaloproteinasas de matriz. De esta forma, el significativo incremento del t-PA en el tejido conectivo gingival inflamado sugiere que los factores de virulencia de las bacterias Gram negativas, como lipopolisacaridos, pueden ser capaces de inducir la expresión del t-PA en el tejido conectivo que luego contribuye a la destrucción del tejido periodontal. ${ }^{9}$

A la vez esto indica que los fibroblastos gingivales pueden ser una fuente del t-PA en el tejido conectivo durante el periodo inflamatorio.

Las posibles fuentes del u-PA en el tejido periodontal incluye macrófagos $\mathrm{y}$ células endoteliales proliferando, que usan el u-PA para su migración. Estas células producen valores incrementados del u-PA cuando son activados apropiadamente in vitro. ${ }^{5,9}$

\section{Inhibidores de los Activadores del plasminógeno en la enfermedad pe- riodontal}

En este caso el PAI que se encuentra en elevadas concentraciones a este nivel es el PAI-2, mientras que el PAI-1 se encuentra limitado al tejido conectivo gingival ${ }^{7}$ ya que su producción está dada por células endoteliales y macrófagos y que su función es el balance de la actividad del t-PA en la sangre. Además, el PAI-1 es extremadamente sensible a la inactivación por oxidantes presentes en el tejido inflamado, por lo tanto es considerado de poca importancia para la inhibición de proteolisis tisular en el FCG ${ }^{12}$, por tal razón su concentración en el FCG es de $25 \mathrm{ng} / \mathrm{ml}$ aproximadamente, lo que equivale a 5 veces su concentración en plasma. Pero esta concentración es solo el $1 \%$ de la concentración del PAI-2 en el FCG. ${ }^{5}$

Por otro lado, PAI-2 es fuertemente sobreexpresado en el epitelio de unión, sulcular y oral, pero nula presencia en el tejido conectivo gingival según su determinación por escalas semicuantitativas. ${ }^{7}$ La concentración media del PAI-2 en el FCG de muestras con periodontitis es de $2300 \mathrm{ng} / \mathrm{ml}$, lo que equivale a casi 10 veces su concentración plasmática en mujeres embarazadas a término, este valor también equivale a casi 500 veces su concentración media en plasma. ${ }^{5}$

Sin embargo, PAI-2 es un inhibidor más lento para t-PA que para u-PA y no se podría excluir que su función también pueda ser inhibir las proteasas bacterianas. ${ }^{7}$ Las células encargadas de su producción son los macrófagos/monocitos $^{1,5,11,12}$, fibroblastos gingivales ${ }^{9} \mathrm{y}$ las células epiteliales más diferenciadas como las capas granulosa y espinosa del epitelio de unión, sulcular y oral. ${ }^{7}$

Los estudios han determinado que el PAI-2 encontrado en el FCG es de elevado peso molecular (EPM) o PAI-2 glicosilado, el cual tendría una función reguladora del t-PA que también es sobreexpresado a este nivel. Este mismo inhibidor se encontró asociado a la membrana celular de queratinocitos, lo cual no afecta su capacidad inhibitoria y así el PAI-2 podría tener una función en la protección contra el ataque proteolítico del epitelio.

Por otro lado, PAI-2 se encuentra también a nivel intracelular pero como PAI-2 no glicosilado o de bajo peso molecular (BPM) lo cual sugiere otro objetivo aparte del t-PA. La función del PAI-2 de BPM intracelular quizás sea de protección contra la apoptosis. ${ }^{15}$

Finalmente, PAI-2 podría ser el más indicado para proteger la gingiva del estrés inflamatorio inhibiendo al t-PA; en este contexto es importante hacer la observación que PAI-2 es mucho más resistente a la oxidación que PAI-1, alfa 1 -inhibidor de proteinasa o que alfa 2-antiplasmina y podría, por lo tanto, ser relativamente resistente a los radicales libres generados en el tejido inflamado. ${ }^{1}$

\section{Relación entre PA y PAI}

En estudios de fibrinolisis tisular, el balance entre PA y PAI es importante para el resultado neto. ${ }^{5} \mathrm{La}$ compara- ción de la proporción molar del t-PA y u-PA para con PAI-2 antes y después del tratamiento periodontal demostró diferencias en varios estudios. ${ }^{5,13} \mathrm{La}$ proporción poco variable entre PAs y PAIs medidas por métodos inmunológicos está en concordancia con los resultados de otra investigación, donde se midió la concentración de plasmina en el FCG con un método funcional y no encontró correlación con el estado periodontal. 5

Esto podría significar un fino mecanismo regulador de los componentes del SAP en los tejidos sanos e inflamados al elevar compensatoriamente los inhibidores de los PA cuando la agresión tisular es activa y disminuirlos cuando la injuria ha cesado ${ }^{5,13}$, pero cabe destacar que existen periodos o estados fisiológicos y patológicos donde este equilibrio es quebrantado y la injuria tisular es clínicamente comprobada como en el caso de gingivitis del embarazo ${ }^{12}$ y las bolsas periodontales reagudizadas. ${ }^{13}$

Además, entre los factores que desencadenan este desequilibrio de proporción entre PA y PAI se encuentran las bacterias periodontales, provocando que los fibroblastos expuestos a lipopolisacaridos (LPS) de patógenos periodontales, en particular Porphyromona gingivalis, generen una proporción de t-PA/PAI-2 mucho más elevado comparado con los fibroblastos expuestos a LPS de E. coli y S. enteritidis. ${ }^{16}$

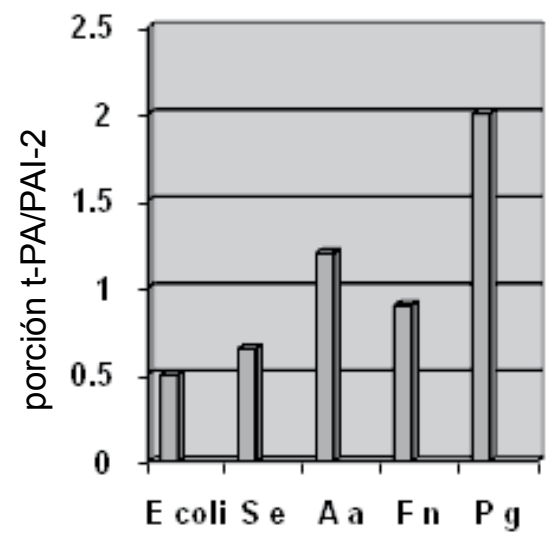

Figura 1. Diferencias en las proporciones entre activador del plasminógeno tipo tisular ( $\mathrm{t}$-PA) e inhibidor del plasminógeno 2 (PAI-2) según su secreción por fibroblastos gingivales expuestos a lipopolisacaridos de E. Coli, S. enteritidis, $A$. actinomycetecomitans, F. nucleatum y $P$. gingivalis. ${ }^{16}$ 


\section{Variación en los niveles de expresión del SAP según el polimorfismo de sus reguladores biológicos}

La respuesta inflamatoria del huésped varía considerablemente entre individuos, los estudios a nivel molecular confirman en muchos casos estas diferencias antes descritas en la clínica. Por consiguiente, el conocimiento que nos brinda el nivel molecular esclarece el panorama y permite un mayor entendimiento de la enfermedad y la posibilidad de prevenirla.

Muchos estudios hasta la fecha se han llevado a cabo para demostrar la participación de las citocinas proinflatorias (IL-1, FNT-alfa, IL-6 e IL-8) en la enfermedad periodontal. ${ }^{17,18,19,20,21}$ Toda la información obtenida sirvió para corroborar que según las concentraciones de las principales citocinas proinflamatorias (IL-1beta y FNT-alfa) se presenta el cuadro clínico de la enfermedad periodontal, en cuanto a la aparición y grado de progresión, más aún a partir del estudio del polimorfismo de la IL-1 ${ }^{22}$ se apreció la utilidad de una prueba para determinar el polimorfismo del mismo ${ }^{23}$, aunque existe todavía controversia al respecto.

A partir de un estudio in vitro ${ }^{24}$ donde las concentraciones de IL-1beta fueron 4 veces superiores para un polimorfismo que en otro, se desarrollaron una serie de estudios para confirmar clínicamente los resultados previamente obtenidos.

Independientemente, de que si los resultados confirmen o no la implicancia de este polimorfismo; lo cierto es que dos de los componentes del SAP, el t-PA y el PAI-2 son sobrerregulados por IL-1beta en fibroblastos gingivales humanos. ${ }^{8,9}$

En conjunto toda la información permite deducir que si el polimorfismo realmente influye en los niveles de IL-1beta, lo cual significaría que existen individuos más susceptibles de presentar el FCG con mayor capacidad fibrinolítica y esto repercutiría en los pacientes con deficiencias en los factores de coagulación donde el coágulo puede ser inestable. Las causas de estas deficiencias podrían ser propias de una enfermedad como en las hepatopatías (cirrosis) o durante el tratamiento con anticoagulantes orales. ${ }^{25}$

\section{Condiciones locales y Sistema Activador del Plasminógeno}

Un estudio más reciente sobre los activadores e inhibidores del Plasminógeno en el fluido crevicular gingival de embarazadas a término y postparto no coincidió con los resultados de Kinnby de 1996 y más bien sugiere que el efecto de las hormonas sexuales en el edema gingival durante el embarazo son más prominentes que los efectos de la acumulación de la placa dental microbiana. ${ }^{26}$

Con respecto a la osteítis alveolar o alveolitis un estudio demostró que la hiperfibrinolisis en la alveolitis se debe a la actividad intensificada de u-PA, por un ligero incremento del $85 \%$, a su vez se demostró que PAI-1 está excepcionalmente sobreexpresado en los tejidos de la zona con alveolitis (hasta 6,7 veces ). Al respecto, de particular interés son los hallazgos en un estudio no con humanos donde la deficiencia de PAI-1 permitía una curación acelerada de heridas de piel. ${ }^{27}$

Buduneli en estudios adicionales sobre los componentes del SAP y su participación en pacientes tratados con Ciclosporina A por transplante renal, debido al crecimiento gingival confirman que el SAP se encuentra alterado aunque sugieren mayores estudios para determinar el verdadero rol de PAI-1 en la condición periodontal de estos pacientes. $^{28}$

Tabla 1. Parámetros fibrinolíticos y de coagulación en pacientes control y cirróticos agrupados según la severidad de la enfermedad hepática, siendo el grupo C el de mayor deterioro de la función hepática. ${ }^{30}$

\begin{tabular}{lcccc}
\cline { 3 - 5 } & Control & \multicolumn{3}{c}{ Pacientes cirróticos (n=53) } \\
\cline { 3 - 5 } & $\mathrm{n}=43$ & $\mathrm{~A}$ & $\mathrm{~B}$ & $\mathrm{C}$ \\
\hline Tiempo de protrombina (s) & 11.3 & 12.4 & 13.3 & 18.3 \\
Factor VII (\%) & 104 & 58.8 & 46.8 & 27.8 \\
Fibrinógeno (mg/dL) & 314 & 260 & 207 & 217 \\
a2-antiplasmina (\%) & 101 & 81 & 76.2 & 55.4 \\
t-PA (ng/mL) & 5.5 & 10.5 & 20 & 23.9 \\
PAl-1 (ng/mL) & 22.4 & 16.6 & 18.5 & 24.1 \\
\hline
\end{tabular}

También encontró un desbalance entre PA y PAI del SAP, donde PAI-2 se encuentra incrementado en casos de periodontitis del fumador. ${ }^{29}$

\section{Condiciones sistémicas y Sistema Activador del Plasminógeno}

Las anormalidades del sistema fibrinolítico encontrados en la cirrosis son complejas y es el resultado de la síntesis defectuosa o disminuida, como es el caso del Inhibidor de la Fibrinolisis Activado por Trombina (TAFI) y alfa 2 antiplasmina, y del clearance alterado como el $\mathrm{t}$-PA, donde este último se encuentra intensificado en la circulación. ${ }^{30}$

Además se corrobora que Deficiencia adquirida de alfa 2 antiplasmina que puede ser visto en pacientes con enfermedad hepática severa debido a su síntesis disminuida. ${ }^{31}$

Durante la sepsis severa todos los componentes del sistema activador del plasminógeno se encuentran incrementados. En el caso del t-PA su neutralización estuvo a cargo del PAI-1, pero se asume que el incremento inusual de u-PA y PAI-2 en plasma, deriva de los leucocitos, a su vez presentes dentro de los depósitos de fibrina, pudiendo influir en la persistencia de fibrina y así contribuir al desarrollo de falla multiorgánica. $^{32}$ 


\section{Referencias bibliográficas}

1. Kruithof et al. Biological and clinical aspects of plasminogen activator inhibitor type2. Blood 1995; 86 (11): 4007-4024.

2. Sprengers et al. Plasminogen Activator Inhibitors. Blood. 1987; 69 (2): 381-387.

3. Kruithof et al. Demonstration of a Fast-Acting Inhibitor of Plasminogen Activators in Human Plasma. Blood. 1984; 64 (4): 907-913.

4. Guyton \& Hall. Tratado de fisiologia medica. McGraw-Hill.1998: 505-516.

5. Kinnby et al. The plaminogen activating system in gingival fluid from adults. Scand J Dent Res. 1994; 102: 334-341.

6. Goodman \& Gilman. Las Bases Farmacológicas de la Terapéutica. Mc Graw Hill. 1996; 1423-1443.

7. Kinnby et al. Localization of plasminogen activators and plasminogen-activator inhibitors in human gingival tissue demonstrated by inmunohistochemistry and in situ hybridization. Arch Oral Biol. 1999; 44: 1027-1034.

8. Xiao et al. Detection of tissue plasminogen activator ( $t-P A)$ and plasminogen activator inhibitor 2 (PAI2) in gingival crevicular fluid from healthy, gingivitis and periodontitis patients. J Clin Periodontol. 2000; 27: 149-156.

9. Xiao et al. Inmunohistochemical demonstration of the plasminogen activator system in human tissues and gingival fibroblasts. J Periodont Res. 1998; 33: 17-26.

10. Pluskota et al. Convergence of the adhesive and fibrinolytic systems: recognition of urokinase by integrin aMb2 as well as by the urokinase receptor regulates cell adhesion and migration. Blood. 2003; 101: 15821590.

11. Kinnby et al. Tissue plasminogen activator (t-PA) and placental plasminogen activator inhibitor (PAI2) in gingival fluid from 8-9 year old children. Scand J Dent Res. 1993; 101: 279-281.
12. Kinnby et al. Aggravation of gingival inflammatory symptoms during pregnancy associated with the concentration of plasminogen activator inhibitor type 2 (PAI-2) in gingival fluid. J Periodontol Res. 1996; 31:271-277.

13. Olofsson et al. Plasminogen activating capacity in gingival fluid from deteriorating and stable periodontal pockets. J Periodont Res. 2002; 37: 60-65.

14. Brown et al. Molecular characterization of plasminogen activators in human gingival crevicular fluid. Arch Oral Biol.1995; 40(9): 839845.

15. Lindberg et al. The localization of the relaxed form of plasminogen activator inhibitor type 2 in human gingival tissue. Histochem Cell Biol. 2001; 116: 447-452.

16. Xiao et al. Effect of lipopolysaccharide from periodontal pathogens on the production of tissue plasminogen activator and plasminogen activator inhibitor 2 by human gingival fibroblasts. J periodontal Res. 2001; 36(1): 25-31.

17. Kjeldsen et al. Bacterial- Stimulated Cytokine Production of Peripherals Mononuclear Cell from patients of various periodontitis categories. J Periodontol. 1995; 66: 139-144.

18. Graves et al. Interleukin-1 and Tumor Necrosis Factor Antagonists Inhibit the Progression of Inflammatory Cell Infiltration Toward Alveolar Bone Experimental Periodontitis. J Periodontol. 1998; 69: 1419-1425.

19. Saito et al. Interlenkin-1 gene expression in macrophages induced by surface protein components of Porphyromonas gingivalis : role of tyrosine Kinases in signal transduction. Oral Microbiol Inmunol. 1997; 12: 135-140.

20. Periodontal Disease as a Potencial Risk Factor for Systemic Diseases. J Periodontol. 1998; 69: 841-850.

21. Dongari-Bagtzoglou et al. Increased Presence of Interleukin-6 (IL-6) and IL-8 Secreting Fibroblast Subpopulations in Adult Periodontitis. J Periodontol. 1998; 69: 899-910.
22. Meisel et al. Smoking and Polymorphisms of the Interleukin-1 Gene Cluster (IL-1alfa, IL-1beta and IL$1 R N)$ in Patients with Periodontal Disease. J Periodontol. 2002; 73: 27-32.

23. Greenstein et al. Clinical utility of a genetic susceptibility test for severe chronic periodontitis. JADA. 2002; 133: 452-459.

24. Pociot et al. A TaqI polymorphism in the human interleukin-1beta (IL-1beta) gene correlates with IL1 beta secretion in vitro. Eur J Clin Invest.1992; 22: 396-402.

25. Méndez Visag. Control del sangrado postoperatorio en pacientes anticoagulados empleando colutorios de acido tranexamico e implicancias de la periodontitis. Odontología Sanmarquina. 2005; 8(1): 14-19.

26. Buduneli et al. Gingival status, crevicular fluid tissue-type plasminogen activator, plasminogen activator inhibitor-2 levels in pregnancy versus post-partum. Australian Dental Journal.2010; 55(3): 292-297.

27. Serrati et al. Plasminogen activators and inhibitors type- 1 in alveolar osteitis. European Journal of Sciences. 2006.114(6): 500-503.

28. Buduneli et al. Plasminogen activators and plasminogen activator inhibitors in gingival crevicular fluid of cyclosporine A- treated patients. J Clin Periodontol. 2004; 31: 556-561.

29. Buduneli et al. Plasminogen activator system in smokers and non-smokers with and without periodontal disease. J Clin Periodontol.2005; 32(14): 417-424.

30. Colucci et al. Deficiency of Thrombin Activatable Fibrinolysis Inhibitor in Cirrhosis is associated with increased plasma fibrinolysis. Hepatology. 2003; 38 (1): 230-237.

31. Cesarman-Maus et al. Molecular mechanism of fibrinolysis. British Journal of Haematology. 2005; 129(3): 307-321.

32. Robbie et al. Plasminogen activator inhibitor 2 and urokinase-type plasminogen activator in plasma and leucocytes in patients with severe sepsis. British Journal of Haematology.2000; 109: 342-348. 